\title{
Reconstrucción quirúrgica de un caso clínico complejo de neuroartropatía de Charcot
}

\author{
Surgical reconstruction of a complex neuroarthopatic charcot foot: a case report \\ Eduardo Simón Pérez ${ }^{1}$, Luke D. Cicchinelli², Javier Pascual Huerta ${ }^{3}$, Jordi Viadé i Julia ${ }^{4}$, \\ Javier Fernández Yagüe ${ }^{5}$, José Ignacio Rodríguez Mateos ${ }^{6}$ y Clarisa Simón Pérez
}

${ }^{1}$ Práctica privada. Hospital Recoletas Felipe II. Valladolid, España. ${ }^{2}$ Práctica Privada. Vigo, España. ${ }^{3}$ Práctica Privada. Clínica del Pie Elcano. Bilbao, España. ${ }^{4}$ Unidad del Pie diabético. Hospital Germans Trias i Puyol. Badalona, Barcelona, España. ${ }^{5}$ Práctica Privada. Tierra Clínica. Madrid, España. ${ }^{6}$ Servicio de Cirugía Plástica, Estética y Reparadora, Hospital Universitario Río Hortega. Valladolid, España. ${ }^{7}$ Servicio de Cirugía Ortopédica y Traumatología, Hospital Clínico Universitario. Valladolid, España

Palabras clave:

Cirugía reconstructiva del pie, pie diabético, neuroartropatía de Charcot, artrodesis, pie varo, artropatía neuropática, pie de Charcot, amputación diabetes mellitus, osteoartropatía.

\section{Resumen}

La neuroartropatía de Charcot es reconocida como una enfermedad destructiva, progresiva y no infecciosa de origen multifactorial en pacientes con diabetes mellitus como principal causa etiológica. Esta destrucción puede ser a diferentes niveles del pie. El presente artículo presenta un caso clínico de un paciente que sufre una deformidad severa rígida en pie varo secundario a neuroartropatía de Charcot tipo II (según clasificación Sanders \& Frykberg) en fase 2 (según clasificación de Eichenholtz) cuya solución de tratamiento propuesto era amputación infracondílea. Se realizó una reconstrucción quirúrgica de la deformidad consistente en alargamiento percutáneo del tendón de Aquiles, tenotomía del tibial posterior, fusión de la columna interna y externa con estabilización mediante placas de bloqueo; también se realizó fusión de la articulación subastragalina y trasposición del extensor común de los dedos y tibial anterior a la base del cuarto metatarsiano. El resultado del caso fue muy bueno y la evolución al año permitió al paciente incorporarse a su actividad diaria. Este artículo pretende mostrar una opción de tratamiento para este tipo de patología con buenos resultados y mostrando una posibilidad de solución a estos pacientes, evitando así la amputación del miembro

\begin{abstract}
Charcot's neuroarthropathy is recognized as a destructive, progressive and non-infectious disease of multifactorial origin in patients with diabetes mellitus as the main etiological cause. This destruction can be at different levels of the foot. This article presents a clinical case of a patient suffering from a severe rigid varus foot deformity secondary to charcot type II neuroarthropathy (according to the Sanders \& Frykberg classification) in phase 2 (according to the Eichenholtz classification) whose recommended treatment solution was infracondylar amputation. A surgical reconstruction of the deformity was performed, consisting of percutaneous lengthening of the Achilles tendon, tenotomy of the posterior tibial, fusion of the internal and external spine with stabilization by means of locking plates, fusion of the subtalar joint and transposition of the common extensor of fingers and tibial anterior to the base of the fourth metatarsal. The result of the case was very good and the evolution after one year experienced the patient joining his daily activity. This article aims to show a treatment option for this type of pathology with good results and to show a possibility of solution to these patients, thus avoiding limb amputation.
\end{abstract}




\section{INTRODUCCIÓN}

El pie de Charcot es una complicación de la diabetes mellitus (DM), que consiste en un proceso degenerativo severo de la estructura osteoarticular del pie que tiene secuelas significativas como movilidad limitada, ulceración, infección, amputación, disminución de la calidad de vida y aumento de la mortalidad global $^{1,2}$. Se estima que entre el 0.1 y el $0.4 \%$ de los pacientes diabéticos desarrollan esta patología que afec-

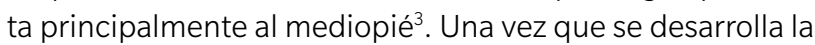
deformidad, la probabilidad de ulceración y amputación se multiplican, reduciendo la calidad de vida de los pacientes ${ }^{4} y$ la esperanza de vida en 14 años aproximadamente ${ }^{5}$.

Podemos diferenciar tres etapas del pie de Charcot $^{6,7}$ : la Etapa 1 o de desarrollo y fragmentación: puede durar entre 3 y 4 meses, en la que existe una gran hiperemia que conduce a la destrucción y fragmentación ósea. El pie tiene un aspecto edematoso, con presencia de rubor y calor que puede confundir con un proceso infeccioso; la Etapa 2 o de coalescencia: dura entre 8 y 12 meses, en el que comienza el proceso reparador. Desaparece el edema, el calor y el rubor. Radiográficamente se aprecia neoformación ósea; la Etapa 3 o de consolidación: hay consolidación de fractura que se puede evidenciar radiológicamente y, en muchas ocasiones, existe deformidad residual.

El tratamiento del pie de Charcot muestra dos tipos de estrategias bien diferenciadas en sus objetivos y métodos de tratamiento, dependiendo de si estamos ante una fase aguda o crónica de la deformidad. En los casos de pie de Charcot agudo está indicado un tratamiento conservador agresivo del pie y miembro inferior mediante descargas absolutas, estabilización y protección como base principal de la terapia, con objeto de evitar la progresión de la deformidad ${ }^{6-8}$. Una vez ya superada la fase aguda e instaurada la deformidad, este tipo de patología supone un reto para el profesional y, en ocasiones, la amputación mayor (por ejemplo la amputación infracondílea) es una de las técnicas que se emplean con relativa frecuencia para solventar estos casos con toda la repercusión médica y socioeconómica que supone para los pacientes y los sistemas satinarios ${ }^{9-11}$.

La cirugía podológica se caracteriza por la especialización de técnicas quirúrgicas en deformidades y patologías del pie. En estos casos, y trabajando de manera multidisciplinar, se pueden obtener resultados óptimos y luchar por el salvamento de la extremidad ${ }^{12}$. El presente artículo presenta un caso clínico de una neuroartropatía de Charcot con afectación de mediopié y retropié en deformidad rígida del pie en varo y con afectación severa a su vez del mediopié y con propuesta previa de amputación infracondílea como solución. El objetivo del presente trabajo es introducir este tipo de técnicas y discutir sus ventajas mediante el presente artículo en la comunidad podológica. El artículo se ha realizado siguiendo la guía CARE (CAse REport Statement) para el reporte y desarrollo de casos clínicos en revistas científicas ${ }^{13}$.

\section{CASO CLÍNICO}

Paciente varón de 46 años que acude al Servicio de Podología de la consulta de la Clínica Recoletas Paracelso (Valladolid) en febrero de 2019, derivado por otro especialista para valoración de tratamiento. El paciente presenta gran deformidad en pie derecho que le impide deambular y desarrollar su trabajo de hostelero, en el que tiene que pasar al menos 10 horas al día de pie.

Entre los antecedentes personales del paciente se destaca la presencia de diabetes mellitus tipo 2 e hipertensión arterial (HTA), ambas de 5 años de evolución. Como complicación crónica de la diabetes, el paciente sufre retinopatía diabética. No presenta alergias medicamentosas conocidas y la medicación del paciente en ese momento era de antidiabéticos orales mediante metformina 850 mg (1-1-1), insulina glarfina 100 unidades/ml (Lantus ${ }^{\circledR}$, Sanofi, España) e ibersartán $300 \mathrm{mg}(0-0-1)$.

Igualmente se destaca que el paciente fue ingresado recientemente por complicaciones en el pie derecho en dos ocasiones (el paciente facilita los informes). La primera fue del 10 al 16 de noviembre de 2018 con juicio diagnóstico de celulitis en miembro inferior derecho, en el que se descartó osteomielitis mediante estudio de RMN y se pautó tratamiento antibiótico (ciprofloxacino $500 \mathrm{mg}$ cada 12 horas durante 14 días) por el edema, calor y tumefacción que presentaba. Este tratamiento no resolvió su inflamación y su cuadro patológico iba progresando, por lo que le condujo al segundo ingreso, que fue cuatro días después (del 20 al 24 de noviembre de 2018) en el que la deformidad y el edema en el miembro inferior derecho habían empeorado y ya eran muy importantes. En este momento, el paciente presentaba una $\mathrm{HbA} 1 \mathrm{c}$ de $11 \%$ y radiográficamente presentaba luxación de Lisfranc. La propuesta de tratamiento entonces fue de amputación infracondílea.

En nuestra consulta, en la exploración se observaba gran deformidad del pie, destacando la posición en varo de la articulación subastragalina de carácter rígido, la deformidad del primer radio en flexión dorsal de carácter rígido y la existencia de una gran prominencia plantar a nivel del cuboides con deformidad en "mecedora" del pie. La movilidad, a nivel de la articulación del tobillo, estaba conservada pero el paciente no podía andar si no se ayudaba con muletas debido a la gran deformidad que presentaba, por lo que la limitación funcional era evidente (Figura 1, Vídeo 1). Se realizó un cribaje neuroisquémico con los siguientes hallazgos: presencia pulsos palpables en arteria pedia $(+++)$ y tibial posterior $(+++)$ del miembro inferior derecho con buen relleno capilar, indicando buena perfusión del pie. El índice Tobillo/Brazo (T/B) en miembro inferior (MI) derecho fue de 0.88. Se comprobaron las sensibilidades algésica (mediante un pin-prick), barestésica (mediante un monofilamento de Semmes-Weinstein) y palestésica (mediante un diapasón) y se concluyó que estaban alteradas tanto la sensibilidad superficial (algésica) como la sensibilidad profunda (barestésica y palestésica). El pacien- 


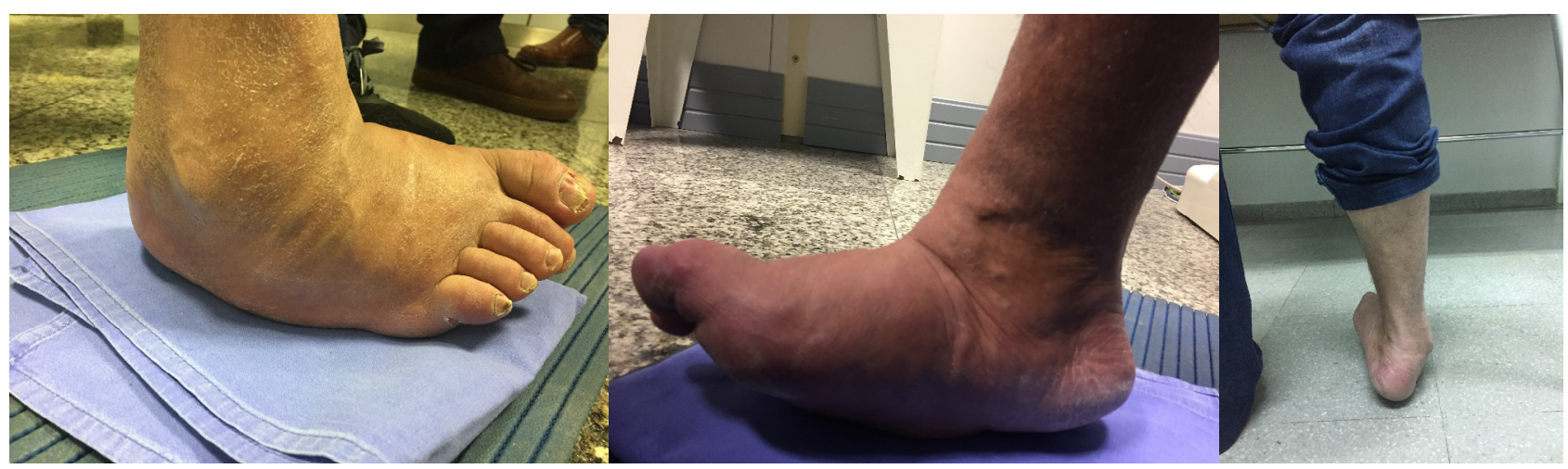

Figura 1. Imágenes preoperatorias del paciente.

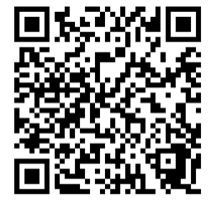

Vídeo 1

te no presentaba ulceraciones, pero tanto en bipedestación estática como en dinámica se observaba hiperpresión en la zona del cuboides por la gran deformidad que presentaba.

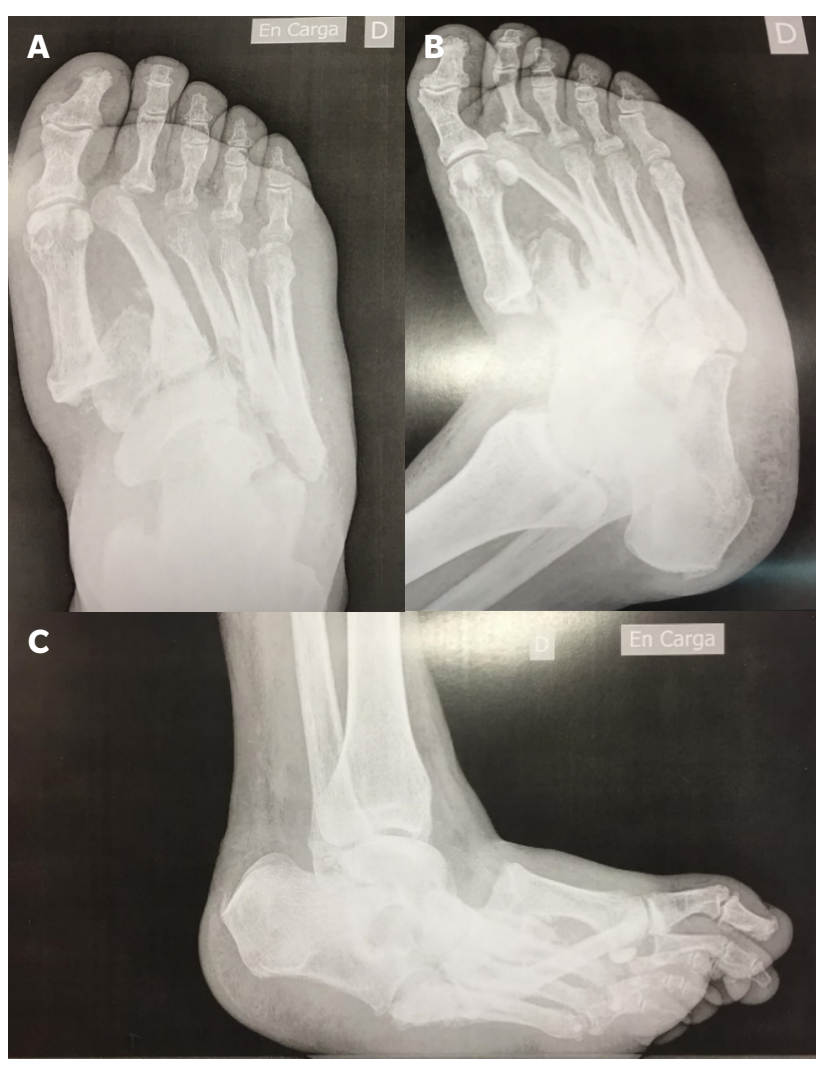

Figura 2. A: radiografía AP en carga. B: Rx oblicua. C: RX lateral en carga.
El paciente aportaba radiografías AP, lateral (en carga) y oblicua en la que se apreciaba luxación severa en articulación de Lisfranc. La base del primer metatarsiano se encontraba totalmente luxada con fractura y luxación de la base del segundo metatarsianos. A nivel de $3 .^{\circ}, 4 .^{\circ}$ y $5 .^{\circ}$ radio había una imagen compatible con destrucción ósea y el cuboides era difícil de identificar con luxación plantar completa por debajo de $3 .^{\circ}, 4 .^{\circ}$ y $5 .^{\circ}$ metatarsianos: la articulación mediotarsiana y el tobillo estaban conservados. La 2. ${ }^{a}$ articulación metatarsofalángica también presentaba luxación completa (Figura 2).

El paciente es diagnosticado de neuroartropatía de Charcot tipo 2 (según clasificación de Sanders \& Frykber ${ }^{1}$ ), en fase 2 (según clasificación de Eichenholtz ${ }^{14}$ ). Se derivó para valoración a la consulta de cirugía vascular para descartar contraindicación quirúrgica y se prescribió la realización de estudio mediante TAC (Figura 3). Desde el servicio de cirugía vascular determinaron que había flujo trifásico a todos los niveles de

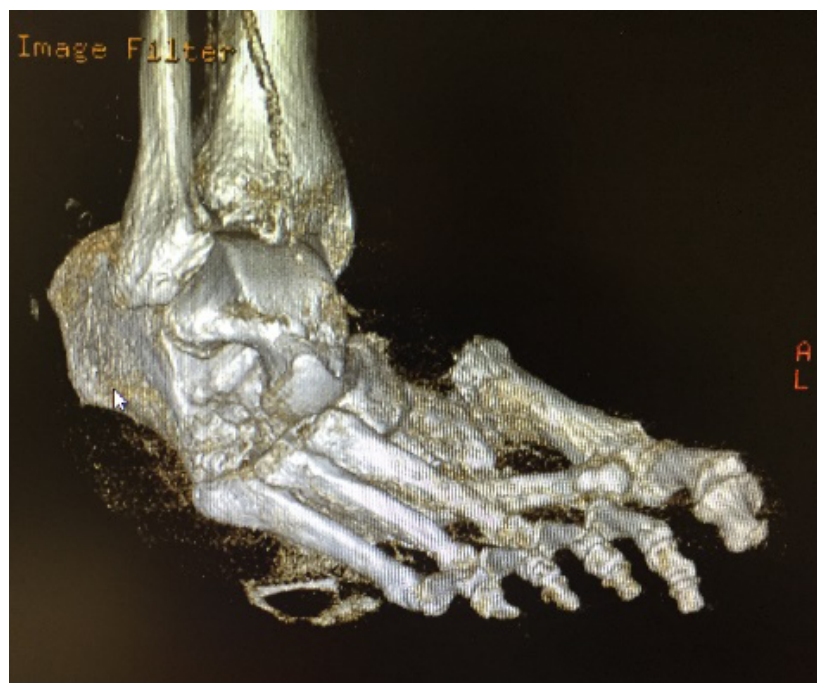

Figura 3. Reconstrucción en 3D del TAC (nótese la lesión de Lisfranc en el $1 .^{\circ}$ y $2 .^{\circ}$ metatarsiano y la ausencia de cuboides que se ha luxado plantarmente. 
tibial anterior, posterior y de las arterias interdigitales, por lo que no existía contraindicación vascular para realizar una intervención quirúrgica en su pie derecho.

Se explicaron al paciente los hallazgos encontrados y se propuso tratamiento quirúrgico de la deformidad consistente en reconstrucción completa mediante alineación del pie con relajación de las fuerzas deformantes del tibial posterior y el tendón de Aquiles y la reducción y estabilización de la luxación que presenta en Lisfranc mediante una artrodesis de dicha articulación y de la articulación subastragalina, que el paciente acepta conociendo los riesgos y complicaciones, siendo las más graves la amputación mayor o la sepsis generalizada.

Se realizó estudio preoperatorio en el que destacaron valores anormales en su analítica de proteína $C$ reactiva (PCR) $1.389 \mathrm{mg} / \mathrm{dl}$ (valor de referencia inferior a 0.50), factor reumatoide $49.4 \mathrm{UI} / \mathrm{ml}$ (valor de referencia inferior a 0.14 ) y vitamina D (25 hidorxicolecalciferol) $15.43 \mathrm{ng} / \mathrm{ml}$ (valor de referencia inferior a 20 se considera déficit). El resto de valores hematológicos, además de la función renal, función hepática, coagulación y $\mathrm{HbA} 1 \mathrm{c}(5.7 \%)$, así como su electrocardiograma no presentaban valores fuera de la normalidad.

Una vez que obtuvimos las imágenes del TAC, se realizó una impresión 3D del mismo para mejor estudio del caso. Se pautó tratamiento previo a la intervención de una solución oral de colecalciferol (vitamina $\mathrm{D}_{3}$ ) $25.000 \mathrm{Ul} / \mathrm{ml}$ (Deltius ${ }^{\circledR}$, Italfarmaco España), 1 cada semana durante dos meses para posteriormente seguir con 1 cada dos semanas durante 2 meses y conjuntamente se pautó calcio (Demilos ${ }^{\circledR}$, italfarmaco, España) 1 cápsula al día durante 4 meses. Ambas medicaciones se pautaron conjuntamente para subir rápidamente los parámetros de vitamina $D$ en el paciente, así como la incorporación del calcio en el tejido óseo antes de la intervención quirúrgica, valor que comprobamos mediante otra analítica la semana previa a la intervención ya programada.

Con fecha de 2 de abril de 2019, y con su vitamina D normalizada, se realizó la intervención quirúrgica mediante sedación consciente, bloqueo raquídeo y hemostasia mediante torniquete quirúrgico a nivel del muslo $(350 \mathrm{~mm} / \mathrm{Hg})$ previa profilaxis antibiótica de cefalosporina (cefalexina $2 \mathrm{~g}$ ).

De manera secuencial, durante la intervención quirúrgica se realizaron los siguientes pasos:

1. Tenotomía percutánea de Aquiles según técnica Hoke ${ }^{15}$, consistente en alargamiento percutáneo del tendón de Aquiles mediante incisión cutánea con tres tenotomías en tercio distal de la pierna; la primera en la parte medial, $2 \mathrm{~cm}$ distal a la inserción en calcáneo, la segunda lateral $2 \mathrm{~cm}$ distal a la primera incisión y la tercera lateral $2 \mathrm{~cm}$ distal a la segunda incisión.

2. Tenotomía completa del tendón tibial posterior mediante incisión medial retromaleolar de 1-2 cm de longitud.

3. Reducción abierta de la deformidad de la columna interna mediante fusión con placa de bloqueo para la columna medial de bajo perfil (3.5 mm - Arthrex, Naples, FL, EE. UU.); para la reducción de la deformidad se realizó incisión desde la articulación astrágalo-esca- foidea hasta la zona diafisaria del primer metatarsiano. Se desinsertó el tendón tibial anterior y se realizaron osteotomías en la base del primer metatarsiano y la cara distal de la primera cuña para reducir en el plano sagital la deformidad de la columna interna. A continuación, se realizó la preparación de las superficies articulares de todas las articulaciones de la columna interna.

4. Previo a la fusión medial se pausó el trabajo medial y se realizó la fusión de la articulación subastragalina fusionada con tornillo de $6.5 \mathrm{~mm}$ tornillo de bajo perfil de 6,7 $\mathrm{mm}$ de diámetro y $75 \mathrm{~mm}$ de longitud (Arthrex, Naples, FL, EE. UU.). La fusión se realizó mediante abordaje abierto a través de la incisión lateral (desde la punta del maleolo externo hasta la articulación de la zona del calcáneo con $4 .{ }^{\circ}$ y $5 .^{\circ}$ metatarsianos (debido a la ausencia del cuboides por su subluxación plantar). Después de preparar y fijar la articulación subastragalina se fusionó la columna medial mediante la placa descrita aprovechando para atravesar con varios tornillos hasta la base del $2 .^{\circ}$ metatarsiano y el mediopié por el escafoides y la cabeza del astrágalo.

5. El siguiente paso fue la reducción abierta de la deformidad de la columna lateral continuando con la incisión lateral utilizada para la fusión subastragalina (Figura 4A); debido a la deformidad existente se realizó extirpación del cuboides (que se utilizó como autoinjerto en la fusión de la columna interna) y se fusionó la columna externa con placa de columna medial de bajo perfil de $3.5 \mathrm{~mm}$ (Arthrex, Naples, FL, EE. UU.) y grapa de compresión después de preparación de todas las superficies. Se suplementó la fijación del mediopié con grapa de compresión y se fijó la columna externa desde el calcáneo hasta la base del $4 .^{\circ}$ metatarsiano. Durante la reparación de la columna externa, y debido a la incapacidad de reducir la deformidad completamente, se reseccionaron las bases de $3 . .^{\circ}, 4 .^{\circ}$ y $5 .^{\circ}$ metatarsianos para poder reducir la deformidad de forma completa y se fijó con una aguja lisa doblada y enterrada para asegurar que la columna externa no se quedara prominente plantarmente ni en varo.

6. Transposición del tibial anterior y el extensor común de los dedos a la base del cuarto metatarsiano mediante tornillo de tenodesis (Biocompsite ${ }^{\circledR}$ y Fiberloop ${ }^{\circledR}$; Arthrex, Naples, FL, EE. UU.). Finalmente, y posterior a la reducción de la deformidad, se realizó sutura por planos utilizando Fiberware ${ }^{\varpi}$ de 2/0 (Arthrex, Naples, FL, EE. UU.) en tejido capsuloligamentoso de la columna medial, sutura absorbible (Vycryl ${ }^{\circledR}$; Johnson \& Johnson, EE. UU.) 3/0 en tejido subcutáneo y monofilamento de Nylon (Ethilon ${ }^{\oplus}$; Johnson \& Johnson, EE. UU.) $3 / 0$ para la sutura de piel junto con puntos de aproximación (Figura 4B). Se realizó vendaje posoperatorio mediante férula completa con venda de fibra de vidrio (Deltacast ${ }^{\oplus}$; BSN Medical, España) a modo de vendaje de Jones modificado ${ }^{8}$. 


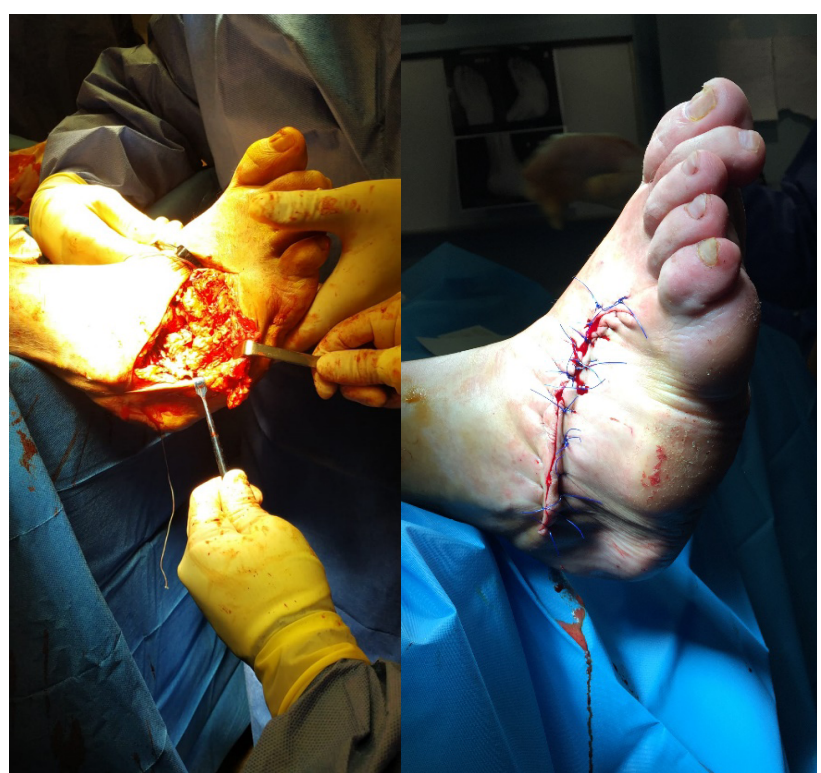

Figura 4. A: abordaje abierto por medio de incisión lateral. B: imagen después de sutura.

El paciente permaneció un día ingresado, y como pautas posoperatorias se recetó tratamiento antibiótico mediante amoxicilina/ácido clavulánico 875/125 (Augmentine ${ }^{\circledR}$, GSK, España) cada 8 horas durante 7 días, dexketoprofeno trometamol (Enantyum ${ }^{\circledast}$, Menarini, España) cada 8 horas durante 14 días, omeprazol $20 \mathrm{mg}$ cada $24 \mathrm{~h}$ durante 14 días y enoxaparina sódica $4000 \mathrm{UI}$ (40 mg)/0,4 ml (Clexane ${ }^{\circledR}$, Sanofi, España) cada 24 horas durante 12 semanas, ya que el paciente permaneció en descarga absoluta durante dos meses, seguido de carga parcial durante un mes.

Las radiografías posoperatorias mostraron buena alineación en todos los planos (Figura 5), excepto en la alineación del segundo radio que continuó mostrando deformidad en las radiografías posoperatorias pero que clínicamente e intraoperatoriamente no pareció relevante ni problemática como para necesitar realineación.

Los puntos se retiraron a los 21 días y se realizaron curas semanales hasta la semana 12 y posteriormente seguimiento en las semanas 14, 16, 18, 20 y 24 , manteniendo las revisiones mensuales hasta el año de la intervención en el que el paciente fue dado de alta. La evolución y las curas no tuvieron incidencias reseñables, el edema posoperatorio fue considerado normal para el tipo de intervención realizada y las heridas cerraron de forma satisfactoria (Figura 6). En el tercer mes se cambió la férula de fibra de vidrio por bota Walker durante un mes y se derivó a la ortopedia para realizar el molde para fabricación de una férula AFO a medida (Figura 7) que el paciente comenzó a utilizar al cuarto mes junto con bota tipo "trekking", y en ese momento el paciente comenzó a incorporar a sus actividades diarias de forma progresiva (sin trabajar). Esta férula se mantuvo durante el primer año después de la

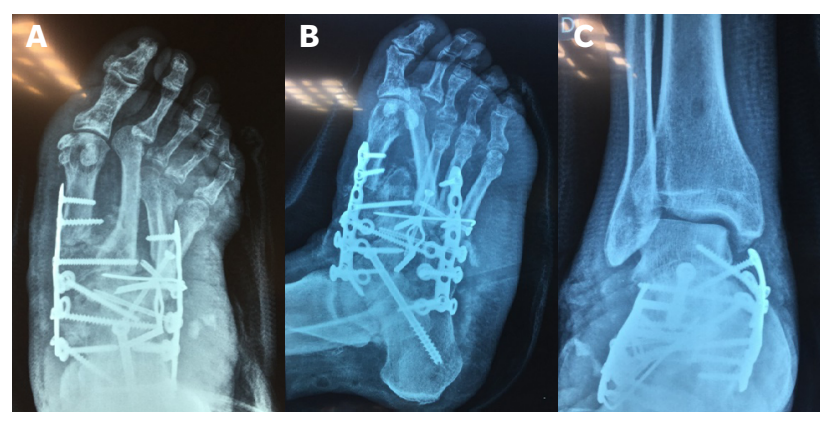

Figura 5. Radiografias posoperatorias. A: AP. B: oblicua. C: AP de tobillo.

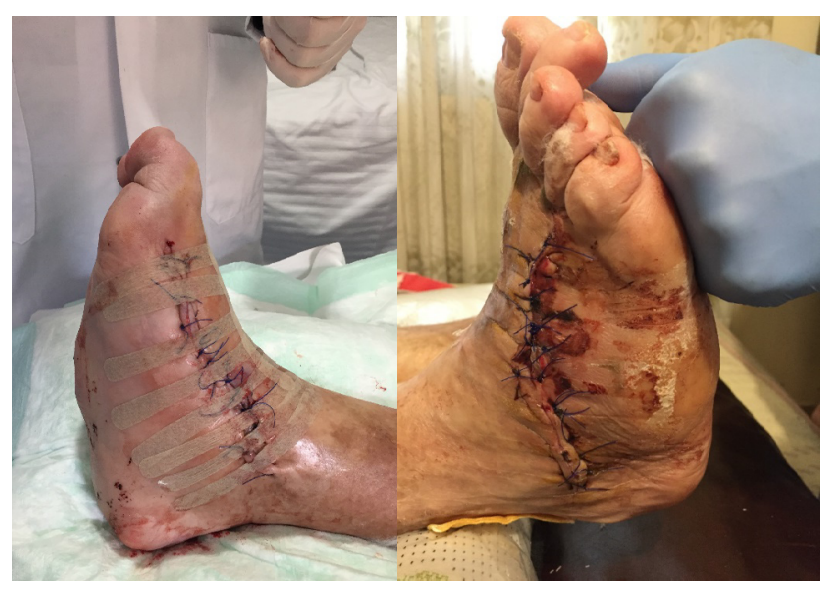

Figura 6. A: imagen a las $24 \mathrm{~h}$ de la intervención. B: imagen a los 15 días de la intervención.

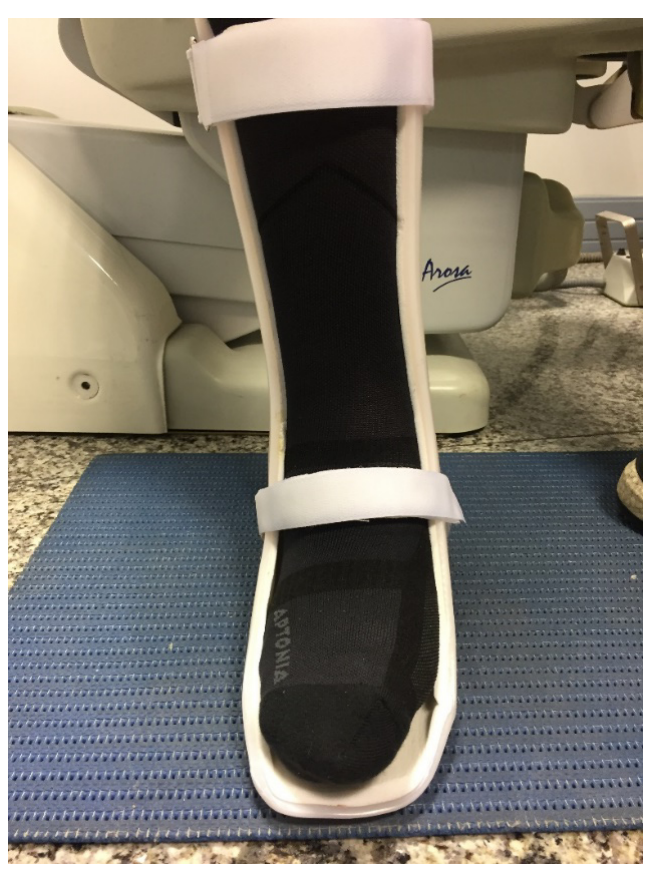

Figura 7. Férula AFO que el paciente comenzó a utilizar a los 4 meses de la intervención. 


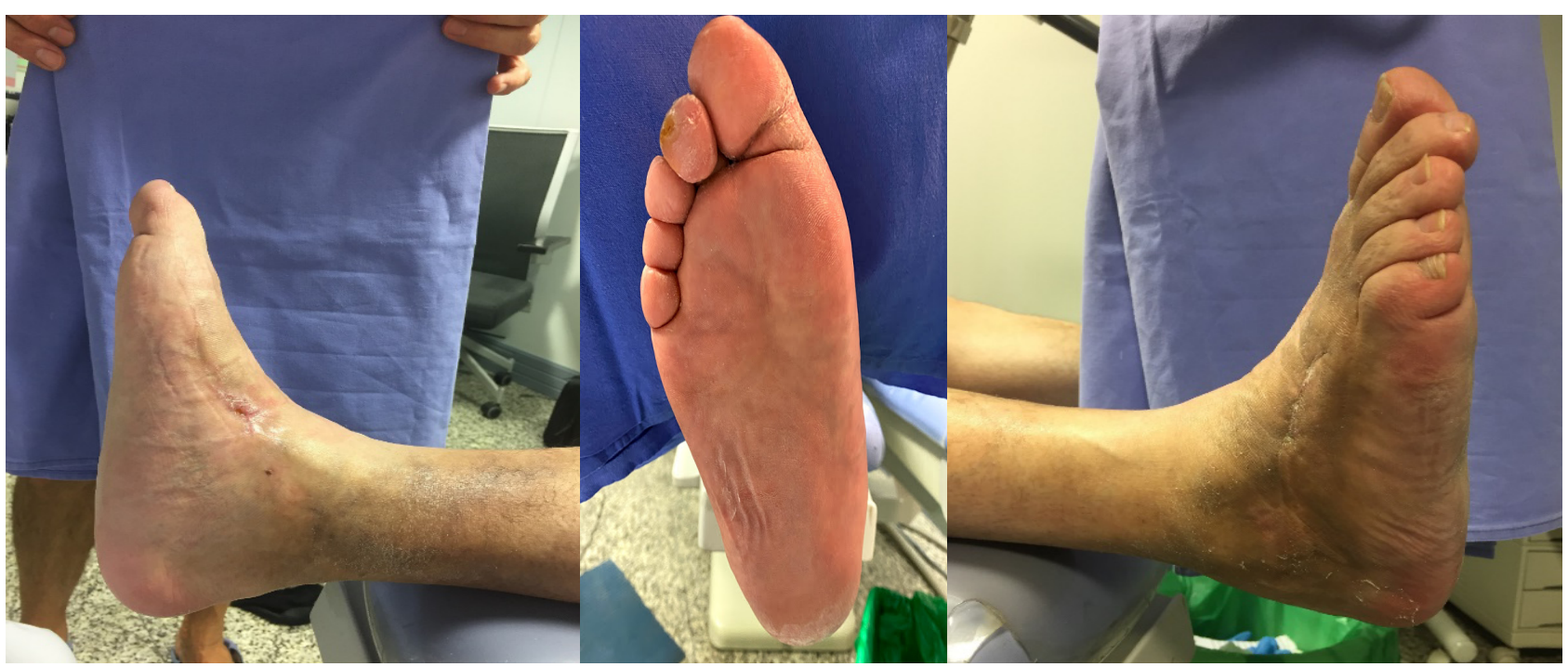

Figura 8. Imágenes posoperatorias al año de la intervención.

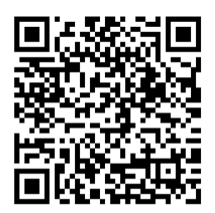

Vídeo 2

intervención. Al año posoperatorio el paciente sigue con su actividad diaria, no ha tenido incidentes reseñables en el pie derecho y se puede observar que el estado actual es óptimo (Figura 8, Vídeo 2).

En el miembro contralateral (izquierda), el paciente, a los 5 meses de la intervención, debutó con una úlcera a nivel del primer dedo que se resolvió mediante descarga selectiva con fieltro adhesivo de $1 \mathrm{~cm}$ y cura tópica con agua purificada, 0,1\% undecilenamidopropil betaína, 0,1\% polihexanida (Prontosan $^{\circledR}, \mathrm{B} /$ Braun, España) y gasas con una capa de contacto antimicrobiana (Sorbact ${ }^{\circledR}$, BSN Medical, España) ${ }^{16,17}$.

\section{DISCUSIÓN}

La neuroartropatía de Charcot continúa siendo actualmente un desafío, tanto diagnóstico como terapéutico, para todos los profesionales y equipos multidisciplinares implicados en el tratamiento del pie diabético. En la actualidad existen discrepancias sobre su etiología y su incidencia ${ }^{3,18}$, con dos teorías principales sobre la aparición de los procesos patológicos que desencadenan en una artropatía de Charcot. La primera es la teoría neurotraumática, que se basa en que el proceso se desencadena debido a un microtraumatismos por repetición o un traumatismo único en un pie insensible ${ }^{1,14}$. La segunda, la teoría neurovascular, defiende que existe una disfunción del sistema autonómico que genera un aumento de flujo sanguíneo y ocasiona un aumento de la resorción ósea y una mayor actividad osteoclástica ${ }^{5,6}$, predisponiendo a una fractura del hueso patológico.

Independientemente de estos factores, hay que señalar que en múltiples ocasiones no existe un diagnóstico correcto de esta patología en sus estadios iniciales, por lo que muchos pacientes sufren un retraso en la aplicación de un tratamiento eficaz que frene la evolución de la deformidad ${ }^{2,3}$. Esto viene motivado por la dificultad existente en realizar un diagnóstico certero en un estadio inicial de la patología (denominado estadio 0) que frecuentemente es confundido con otros procesos inflamatorios como celulitis, gota, trombosis venosa profunda o un simple esguince de tobillo ante la ausencia de deformidades clínicas o radiológicas ${ }^{20,21}$. En el caso clínico presentado en el presente artículo, el paciente sufrió un proceso inflamatorio agudo en noviembre de 2018 por el que fue ingresado en dos ocasiones consecutivas y que posiblemente pudiera corresponder con una presentación aguda de neuroartropatía de Charcot en estadio 0 , que fue inicialmente tratada como un proceso de celulitis (sin descarga de la extremidad) y que inmediatamente después evolucionó a una fractura patológica con deformidad severa del pie. En la opinión de los autores, este es un proceso muy común y es importante tener en cuenta la sospecha de neuroartropatía de Charcot ante la presencia de cuadros inflamatorios agudos del pie y tobillo en pacientes neuropáticos (aun sin presencia de confirmación radiológica), con la idea de pautar un tratamiento mediante descarga agresiva del miembro inferior y evitar así la progresión hacia deformidades más severas del pie, y que posteriormente complicarán mucho el tratamiento y pronóstico del miembro inferior ${ }^{3,21,22}$.

El tratamiento de la artropatía de Charcot en fase aguda consiste en la inmovilización y descarga agresiva del miembro inferior. Este tratamiento puede durar de 8 a 16 semanas, 
seguido de un periodo variable de carga protegida que puede llegar a prolongarse hasta 10 meses $^{7,23}$. Por su parte, en los casos de Charcot crónico en los que ya se ha superado la fase aguda y en los que existe deformidad del pie ya instaurada, el objetivo del tratamiento consiste en conseguir una marcha plantígrada y evitar la formación de ulceraciones en el pie, dando la máxima funcionalidad al paciente para que pueda realizar su vida normal. En estos estadios las opciones de tratamiento pueden ser tanto conservadoras como quirúrgicas con objeto de acomodar el pie a una posición plantígrada que evite la formación de ulceraciones o corregir la posición del pie aportando más funcionalidad y evitando igualmente la formación de ulceraciones en el futuro ${ }^{24}$. Ante la presencia de ulceración, están indicadas medidas conservadoras, como las descargas con yesos de contacto total, botas tipo "Walker" y plastic cast, que es una botina de vendas sintéticas de fibra de vidrio hecha a medida mediante una técnica rápida y sencilla para descarga total en úlceras del pie diabético ${ }^{25}$. Una vez que la ulceración ha sido resuelta y la deformidad no representa un gran impedimento al paciente (el paciente presenta un apoyo del pie plantígrado), se puede realizar una adaptación de plantillas y/o calzado a medida ${ }^{12,26}$.

Por su parte, la decisión de realizar intervención quirúrgica en estos pacientes viene motivada por varios factores, siendo los más importantes la presencia de ulceraciones recurrentes por deformidades o prominencias óseas no reducibles o no acomodables de forma conservadora o la presencia de un pie totalmente afuncional no plantígrado, como es el caso clínico presentado. En este tipo de casos la propuesta de amputación infracondílea es un tratamiento comúnmente utilizado como solución definitiva de la deformidad ${ }^{27}$. Sin embargo, la reconstrucción quirúrgica de la deformidad puede suponer una alternativa terapéutica viable en este tipo de casos, evitando la amputación del miembro inferior. Los procedimientos quirúrgicos reconstructivos en estos casos son complejos y durante la reconstrucción se pueden utilizar dispositivos de fijación interna y externa para la reparación y estabilización del pie que incluyen, tornillos de compresión, grapas y agujas de kirschner. Los procedimientos realizados dependen del tipo y la gravedad de la afección. La cirugía puede involucrar cualquier parte del pie y el tobillo, y puede involucrar también la reparación y/o reequilibrio de las fuerzas deformantes en tendones, articulaciones, tejidos capsuloligamentosos o piel. Las cirugías correctivas a veces se pueden realizar de forma ambulatoria con técnicas mínimamente invasivas y el tiempo de recuperación va a depender del tipo de afección a tratar ${ }^{28}$.

A pesar de todo esto, no hay evidencias científicas de resultados óptimos en cuanto al tratamiento quirúrgico de la artropatía de Charcot una vez la enfermedad ya se ha cronificado ${ }^{29}$. Todo esto puede ser debido al alto riesgo de desarrollar complicaciones secundarias a la cirugía de estos pacientes, tales como: infección perioperatoria, dehiscencia de la herida, fallo de material de osteosíntesis, fracaso de la corrección, pseudoartrosis o nuevo proceso de artropatía de
Charcot. No obstante, en los casos en los que el paciente presente una limitación en su actividad diaria por la deformidad desarrollada o una deformidad totalmente afuncional, estaría indicada una intervención para restaurar esta deformidad. Esto representa aproximadamente entre el 25 y el $50 \%$ de los pacientes que han desarrollado una neuroartropatía de Char$\cot ^{27,29}$. A pesar de las series de casos presentadas en este sentido, son necesarios más estudios que intenten arrojar luz sobre la conveniencia de intervenciones quirúrgicas y tipo de ellas en este tipo de pacientes ${ }^{28-31}$.

Este caso presenta unas peculiaridades únicas en su reconstrucción quirúrgica que lo hace especialmente complejo. Comprobando la buena alineación del tobillo se intentó rotar el pie alrededor del astrágalo ${ }^{32}$, por eso se relajaron primero las partes blandas para disminuir las fuerzas deformantes que contribuían a la deformidad (tendón de Aquiles y tibial posterior) para continuar con la descompresión de la deformidad en la columna medial y con la fusión de la articulación subastragalina. Esta fusión de la articulación subastragalina ayudó a tener una referencia de la posición neutra del pie que se quería conseguir para posteriormente acomodar la posición de la articulación de Lisfranc mediante la fusión de la columna externa e interna (con abordajes medial y lateral) y finalizar con la transposición tendinosa del tibial anterior y extensor largo común de los dedos a la base del cuarto metatarsiano, consiguiendo así una flexión dorsal del pie con un vector de tracción más lateralizado a la posición del eje de la articulación subastragalina, ayudando así a la corrección de la deformidad en varo del paciente. No obstante, entendemos que el caso es de una complejidad importante en el que destacamos la utilización de abundante material de osteosíntesis mediante placa medial y lateral que ayuda a estabilizar la deformidad del pie a modo de "peine" con tornillos intercalados y que refuerzan la estabilidad de la construcción creada para evitar el colapso con la carga posterior del paciente.

En el proceso posoperatorio de estos casos juega un papel muy importante para la correcta solución del mismo en el contexto de la descarga y deambulación progresiva parcial. Es importante en estos casos un seguimiento cercano del paciente, ya que los pacientes diabéticos que han desarrollado complicaciones crónicas de la diabetes tienen una mayor predisposición a las complicaciones posquirúrgicas $24,27,28,33,34$. En este caso el paciente se mantuvo en descarga absoluta del pie intervenido durante 12 semanas para asegurar una consolidación ósea de manera efectiva y en la posición que se había conseguido en la cirugía, ya que conocemos estudios que muestran el retraso de consolidación ósea de estos pacientes comparados a pacientes sin diabetes ${ }^{35-38}$. La idea de fondo al tratar estos pacientes es prácticamente "doblar" todos los aspectos de la cirugía con respecto a un paciente no diabético para protegerlo lo más posible, por lo que se utiliza más material de osteosíntesis y se inmoviliza durante más tiempo. Posterior a las 12 semanas de descarga, el paciente comenzó a utilizar una bota tipo Walker durante un mes y posteriormente una férula $\mathrm{AFO}^{39}$ al ser un aparato efi- 
ciente y cómodo para el tratamiento ortésico después de la cirugía reconstructiva ${ }^{40}$, que aún sigue utilizando, teniendo como propósito evitar que el trauma de la intervención pueda provocar otro proceso de neuroartropatía de Charcot ${ }^{1,14}$. Esta férula AFO ayuda a proporcionar un reparto de cargas más homogéneo ${ }^{41,42}$ y una protección mayor para que el paciente desarrolle su actividad diaria y labora| ${ }^{33,43}$.

Finalmente, es importante también señalar la importancia que tuvo en este caso el nivel de vitamina D normalizado a la hora de intervenir este tipo de casos, y más en estos pacientes, ya que influye en el proceso de la osteosíntesis en la disminución del riesgo no unión, infección e incluso de la depresión, factor a tener en cuenta tanto por el largo posoperatorio como por la propia patología del paciente ${ }^{44,45}$.

En conclusión, la neuroartropatía de Charcot supone un desafío único para todos los profesionales implicados en el tratamiento del pie diabético. El diagnóstico de la neuroartropatía de Charcot en sus estadios iniciales requiere un fuerte índice de sospecha y el reconocimiento y la intervención temprana pueden limitar la progresión de la deformidad ${ }^{46}$. El manejo conservador debe iniciarse lo antes posible y de manera agresiva para minimizar los efectos devastadores, que a menudo se ven con esta afección. Cualquier retraso en la terapia puede resultar en una deformidad severa del pie y el tobillo, en la cual los métodos tradicionales no quirúrgicos por sí solo pueden resultar insuficientes. Estas deformidades pueden conducir a ulceraciones y, en última instancia, progresar a la amputación de la extremidad inferior. La corrección y estabilización quirúrgica puede ser un método efectivo para prevenir una mayor deformidad y recurrencia de ulceraciones. Por ello, ante deformidades tan complejas como la presentada en el presente artículo, se aconseja contemplar una solución mediante una reconstrucción quirúrgica. Es fundamental la comprensión de la deformidad desde el punto de vista biomecánico para poder entender cómo se comporta el pie en ese momento y cómo queremos que se comporte después de la intervención, intentando fusionar las deformidades presentes y estabilizar la estructura del pie con un constructo de fijación interna rígida muy estable. La importancia de valorar y tratar al paciente como un caso único ${ }^{31}$, conseguir una mayor habilidad técnica, conocimiento y avances en la fijación es el objetivo para conseguir que estas deformidades sean cada vez más manejables. En el futuro, esta experiencia debería instigar a la comunidad, en general, a crear protocolos basados en evidencia y en el trabajo multidisciplinar para acercarnos un poco más al éxito en el tratamiento de estas deformidades complejas del pie ${ }^{46,47}$.

\section{CONFLICTOS DE INTERESES}

Los autores no presentan conflictos de intereses relevantes con el presente artículo.

\section{FINANCIACIÓN}

No presenta.

\section{BIBLIOGRAFÍA}

1. Rogers LC, Frykberg RG, Armstrong DG, Boulton AJ, Edmonds M, Van GH, et al. The Charcot foot in diabetes. Diabetes Care. 2011;34(9):21239. DOI: $10.2337 / \mathrm{dc} 11-0844$.

2. Labovitz JM, Shofler DW, Ragothaman KK. The impact of comorbidities on inpatient Charcot neuroarthropathy cost and utilization. J Diabetes Complications. 2016;30(4):710-5. DOI: 10.1016/j.jdiacomp.2016.01.004.

3. Asunción J, Valcarcel PA, Poggio D. Neuroartropatía de Charcot. $1^{\text {a }}$ Parte. Revista Pie Diabético. 2011;12:6-18.

4. Gazis A, Pound N, Macfarlane R, Treece K, Game F, Jeffcoate W. Mortality in patients with diabetic neuropathic osteoarthropathy (Charcot foot). Diabet Med. 2004;21(11):1243-6. DOI: 10.1111/j.14645491.2004.01215.x

5. Van Baal J, Hubbard R, Game F, Jeffcoate W. Mortality associated with acute charcot foot and neuropathic foot ulceration. Diabetes Care. 2010;33(5):1086-9. DOI: 10.2337/dc09-1428.

6. Armstrong DG, Lavery LA. Acute Charcot's arthropathy of the foot and ankle. Phys Ther. 1998;78(1):74-80. DOI: 10.1093/ptj/78.1.74.

7. Pinzur MS, Lio T, Posner M. Treatment of Eichenholtz stage I Charcot foot arthropathy with a weight-bearing total contact cast. Foot Ankle Int. 2006;27(5):324-9. DOI: 10.1177/107110070602700503.

8. YU GV, Schubert EK, Khoury WE. The Jones compression bandage: Review and clinical applications. J Am Podiatr Med Assoc. 2002;92(4):221-31. DOI: 10.7547/87507315-92-4-221.

9. Boulton AJM. The diabetic foot. Med (United Kingdom). 2019;47(2):1005. DOI: 10.1016/j.mpmed.2018.11.001.

10. Fard AS, Esmaelzadeh M, Larijani B. Assessment and treatment of diabetic foot ulcer. Int J Clin Pract. 2007;61(11):1931-8. DOI: 10.1111/j.1742-1241.2007.01534.x.

11. Crawford F, Mccowan C, Dimitrov BD, Woodburn J, Wylie GH, Booth E, et al. The risk of foot ulceration in people with diabetes screened in community settings: Findings from a cohort study. QJM. 2011;104(5):40310. DOI: 10.1093/qjmed/hcq227.

12. Viadé J. Pie Diabético. Guia Practica Para La Prevencion, Evaluacion y Tratamiento. 1. a ed. Madrid: Ed. Panamericana; 2006.

13. Gagnier JJ, Riley D, Altman DG, Moher D, Sox H, Kienle GS. The CARE guidelines: consensus-based clinical case reporting guidelines development. Dtsch Arztebl Int. 2013;110(37):603-8. DOI: 10.3238/arztebl.2013.0603.

14. Van Der Ven A, Chapman CB, Bowker JH. Charcot neuroarthropathy of the foot and ankle. J Am Acad Orthop Surg. 2009; 17(9):562-71. DOI: 10.5435/00124635-200909000-00003

15. Hatt rn, Lamphier TA. Triple hemisection: a simplified procedure for lengthening the Achilles tendon. N Engl J Med. 1947;236(5):166-9. DOI: 10.1056/NEJM194701302360502.

16. Dorresteijn JAN, Kriegsman DM, Assendelft WJJ, Valk GD. Patient education for preventing diabetic foot ulceration. Cochrane Database Syst Rev. 2012;(10):CD001488. DOI: 10.1002/14651858.CD001488. pub4.

17. Mcinnes A, Jeffcoate W, Vileikyte L, Game F, Lucas K, Higson N, et al. Foot care education in patients with diabetes at low risk of complications: A consensus statement. Diabet Med. 2011;28(2):162-7. DOI: 10.1111/j.1464-5491.2010.03206.x.

18. Botek G, Anderson MA, Taylor R. Charcot neuroarthropathy: An often overlooked complication of diabetes. Cleve Clin J Med. 2010;77(9):5939. DOI: 10.3949/ccjm.77a.09163.

19. Baumhauer JF, O'Keefe RJ, Schon LC, Pinzur MS. Cytokine-induced osteoclastic bone resorption in charcot arthropathy: An immunohistochemical study. Foot Ankle Int. 2006;27(10):797-800. DOI: 10.1177/107110070602701007.

20. Chantelau E. The perils of procrastination: Effects of early vs. delayed detection and treatment of incipient Charcot fracture. Diabet Med. 2005;22(12):1707-12. DOI: 10.1111/j.1464-5491.2005.01677.x.

21. Yu GV, Hudson JR. Evaluation and treatment of stage 0 Charcot's neuroarthropathy of the foot and ankle. J Am Podiatr Med Assoc. 2002;92(4):210-20. DOI: 10.7547/87507315-92-4-210. 
22. Schon LC, Marks RM. The management of neuroarthropathic fracture-dislocations in the diabetic patient. Orthop Clin North Am. 1995;26(2):375-92.

23. Petrova NL, Edmonds ME. Conservative and Pharmacologic Treatments for the Diabetic Charcot Foot. Clin Podiatr Med Surg. 2017;34(1):15-24. DOI: 10.1016/j.cpm.2016.07.003.

24. Gil J, Schiff AP, Pinzur MS. Cost comparison: Limb salvage versus amputation in diabetic patients with charcot foot. Foot Ankle Int. 2013;34(8):1097-9. DOI: 10.1177/1071100713483116.

25. Viadé Julià J, Huguet Vidal T. Botina con fibra de vidrio para descarga total en úlceras del pie diabético. Revista Pie Diabético. 2010;10:5-9.

26. Viadé Julià J, Carbó Perez J. Neuropatía de Charcot (II). Tratamiento ortésico. Revista Pie Diabético. 2011;13:2-9.

27. Schneekloth BJ, Lowery NJ WD. Charcot Neuroarthropathy in Patients With Diabetes: An Updated Systematic Review of Surgical Management. J Foot Ankle Surg. 2016;55(3):586-90. DOI: 10.1053/j. jfas.2015.12.001.

28. Varma AK. Reconstructive foot and ankle surgeries in diabetic patients. Indian J Plast Surg. 2011;44(3):390-5. DOI: 10.4103/0970-0358.90806.

29. Ríos Ruh JM, Viadé Juliá J, Reverter Calatayud JL, Santamaría Fumas A, Pérez MRJMS. Tratamiento de las lesiones de mediopié en la neuropatía de Charcot. Revista Pie Diabético. 2016;28:13-24.

30. Pinzur MS. Surgical treatment of the Charcot foot. Diabetes Metab Res Rev. 2016;32(Suppl 1):287-91. DOI: 10.1002/dmrr.2750.

31. Cicchinelli LD. The Importance of $N=1$. J FootAnkle Surg. 2012;51(3):27980. DOI: 10.1053/j.jfas.2012.03.004.

32. Cicchinelli LD. Cirugía del pie plano: una visión personal. Rev Esp Podol. 2018;29(1):49-59. DOI: 10.20986/revesppod.2018.1516/2018.

33. Pinzur MS. Current concepts review: Charcot arthropathy of the foot and ankle. Foot Ankle Int. 2007;28(8):952-9. DOI: 10.3113/ FAl.2007.0952.

34. Wukich DK, Lowery NJ, McMillen RL, Frykberg RG. Postoperative infection rates in foot and ankle surgery: A comparison of patients with and without diabetes mellitus. J Bone Jt Surg - Ser A. 2010;92(2):287-95. DOI: $10.2106 / J B J S . I .00080$.

35. Gandhi A, Liporace F, Azad V, Mattie J, Lin SS. Diabetic Fracture Healing. Foot Ankle Clin. 2006;11(4):805-24. DOI: 10.1016/j.fcl.2006.06.009.
36. Follak N, Klöting I, WolfE, Merk H. Delayed remodelling in the early period of fracture healing in spontaneously diabetic BB/OK rats depending on the diabetic metabolic state. Histol Histopathol. 2004; 19(2):473-86. DOI: $10.14670 / \mathrm{HH}-19.473$.

37. Loder RT. The influence of diabetes mellitus on the healing of closed fractures. Clin Orthop Relat Res. 1988;(232):210-6. DOI: 10.1097/00003086-198807000-00028.

38. Boddenberg U. Healing time of foot and ankle fractures in patients with diabetes mellitus: Literature review and report on own cases. Zentralbl Chir. 2004;129(6):453-9. DOI: 10.1055/s-2004-832398.

39. Wukich DK, Kline AJ. The management of ankle fractures in patients with diabetes. J Bone Jt Surg - Ser A. 2008;90(7):1570-8. DOI: 10.2106/ JBJS.G.01673.

40. Koller A, Meissner SA, Podella M, Fiedler R. Orthotic Management of Charcot Feet After External Fixation Surgery. Clin Podiatr Med Surg. 2007;24(3):583-99. DOI: 10.1016/j.cpm.2007.03.012.

41. Bregman DJJ, Van Der Krogt MM, De Groot V, Harlaar J, Wisse M, Collins $\mathrm{SH}$. The effect of ankle foot orthosis stiffness on the energy cost of walking: A simulation study. Clin Biomech. 2011;26(9):955-61. DOI: 10.1016/j.clinbiomech.2011.05.007.

42. Ploeger HE, Waterval NFJ, Nollet F, Bus SA, Brehm MA. Stiffness modification of two ankle-foot orthosis types to optimize gait in individuals with non-spastic calf muscle weakness-A proof-of-concept study. J Foot Ankle Res. 2019;12:41. DOI: 10.1186/s13047-019-0348-8.

43. Cortés Barragán JM. Biomecánica en las AFOS. AFO podiátricas. Rev Española Podol. 2012;23(5):184-94.

44. Iglar PJ, Hogan KJ. Vitamin D status and surgical outcomes: A systematic review. Patient SafSurg. 2015;9:14. DOI: 10.1186/s13037-015-0060-y.

45. Cuomo A, Giordano N, Goracci A, Fagiolini A. Depression and Vitamin D Deficiency: Causality, Assessment, and Clinical Practice Implications. Neuropsychiatry (London). 2017;7(5):606-14. DOI: 10.4172/neuropsychiatry. 1000255.

46. Frykberg RG, Belczyk R. Epidemiology of the Charcot Foot. Clin Podiatr Med Surg. 2008;25(1):17-28. DOI: 10.1016/j.cpm.2007.10.001.

47. Burns PR, Wukich DK. Surgical Reconstruction of the Charcot Rearfoot and Ankle. Clin Podiatr Med Surg. 2008;25(1):95-120. DOI: 10.1016/j. cpm.2007.10.008. 